\title{
Coronary Artery Mapping: A Method for Three-Dimensional Reconstruction of Epicardial Anatomy
}

\author{
Christopher M. Speidel, Ronald K. Walkup, Dana R. Abendschein, Joseph L. Kenzora, and \\ Michael W. Vannier
}

\begin{abstract}
Evaluation of coronary anatomy with conventional coronary angiography requires visual integration of multiple images from different viewing orientations to generate a mental interpretation of three-dimensional (3D) structure. The epicardial surface is, in many ways, analogous to the earth's surface topography and may be effectively depicted using cartographic methods. To show coronary anatomy visualized as topographic maps, we used cartographic projection methods to analyze the coronary vessels of a canine heart after immediate postmortem injection with a radio-opaque gelatinous solution. A volumetric image data set was obtained with $x$-ray spiral computed tomography. The principal axis of the image volume was calculated and the image volume reformatted to a reference coordinate system defined by the principal axis as the ordinate. A cylindrical projection map of the epicardial surface was created using a maximum-intensity projection volume-rendering technique. After converting the Cartesian reference coordinate system to a polar coordinate system, additional mapping projections from user-defined orientations were generated. The results show that interpretative difficulties of coronary angiography may be diminished by generating 3D maps of coronary anatomy using volumetric datasets acquired noninvasively and displayed with cartographic methods.

Copyright 1995 by W.B. Saunders Company
\end{abstract}

KEY WORDS: Coronary artery disease, angiography, cartography, spiral computed tomography.

$\mathbf{A}^{\mathrm{c}}$ CCURATE interpretation of conventional coronary angiograms requires visual integration of numerous sequential images from different reviewing orientations to generate a three-dimensional (3D) mental representation of the coronary anatomy. This subjective process of visual interpretation can result in variable results regarding the observed object's size and shape. Furthermore, the comprehension of this complex anatomy is often hampered by superimposed semitransparent structures or opaque scene components. Despite these interpretive difficulties, invasive coronary angiography remains the only clinical method available to visualize the epicardial coronary anatomy.

The noninvasive evaluation of coronary arteries with magnetic resonance angiography has been described. ${ }^{1,2}$ These initial attempts have met with limited success because of respiratory and cardiac motion artifacts. Recently, a fatsuppressed, breath-hold, electrocardiogramgated gradient-echo pulse sequence has been described with which the major epicardial arteries, as well as significant stenoses and occlusions, can be identified. ${ }^{3}$ Similar to conventional coronary angiography, the interpretation of magnetic resonance coronary angiograms requires mental integration of multiple $2 \mathrm{D}$ images to formulate a visual $3 \mathrm{D}$ interpretation of the epicardial coronary anatomy, ${ }^{4}$ and is likewise subject to interpretative difficulties because of overlapping semitransparent or opaque scene components.

The 3D characteristics of geographic structures can be analyzed and displayed in two dimensions using map projections or cartography. ${ }^{5}$ Cartography relies on the projection of surface structures onto a superimposed transparent object (eg, a cone or a sphere). In some instances, the surface of this object is then "unwrapped" about the longitudinal principal axis that corresponds to the object's major axis, and displayed as a 2D map. ${ }^{6}$ The principal axes correspond to the coordinate triad that passes through the centroid of the object, and are intrinsic properties of the object's shape. The use of principal moments and axes of inertia for our study was motivated by the need to define a local coordinate system for the heart, independent of the tomographic imaging device. The principal axes of inertia satisfy this requirement and have long been used for this purpose ${ }^{7}$ and for the registration of image volumes. ${ }^{8}$

The epicardial surface contains features analogous to earth topography. The spatial characteristics of the coronary arterial tree, for example, are analogous to geographic struc-

From the Department of Medicine, Washington University School of Medicine; and the Mallinckrodt Institute of Radiology, St. Louis, $M O$.

Address reprint requests to Michael $W$. Vannier, $M D$, Mallinckrodt Institute of Radiology, 510 S Kingshighway Blvd, St Louis, MO 63110.

Copyright $\odot 1995$ by W.B. Saunders Company

0897-1889/95/0801-0005\$3.00/0 
tures (eg, rivers, roads). ${ }^{9}$ Accordingly, cartographic methods developed to analyze geographic structures and display their 3D characteristics as $2 \mathrm{D}$ maps may be used to analyze and display anatomic structures.

Presented here are the results of two 3D reformation techniques of postmortem canine coronary anatomy using ANALYZE (Mayo Clinic, Rochester, $\mathrm{MN})^{10}$ and the software development package, IDL (Research Software Inc, Denver, CO). The principal axis of the canine heart was identified and the object reformatted to a reference coordinate system defined by the principal axis as the ordinate. The surface coordinates describing the epicardial anatomy were then displayed as 2D surface maps.

\section{MATERIALS AND METHODS}

\section{Materials}

An iodinated solution was prepared for injection into the coronary arteries by dissolving gelatin (Knox Gelatin, Inc, Englewood Cliffs, NJ) in preheated iodinated intravascular contrast (Conray 30, Mallinckrodt Medical, St Louis, MO). Food coloring (FD\&C No. 1) was added to the solution so that the extent of coronary perfusion could be determined visually.

\section{Animal Preparation}

All procedures were conducted according to the guiding principles of the American Physiological Society. A male mongrel dog weighing $\sim 20 \mathrm{~kg}$ was premedicated with subcutaneous acepromazine maleate $(0.5 \mathrm{mg} / \mathrm{kg})$ and atropine sulfate $(0.04 \mathrm{mg} / \mathrm{kg})$ after an overnight fast. Anesthesia was induced with intravenous thiopental $(15 \mathrm{mg} / \mathrm{kg})$. The lungs were mechanically ventilated and anesthesia was maintained with halothane (1.4\%). An intravenous catheter was placed in the external jugular vein. At the completion of a femoral artery thrombosis experiment being performed at the medical center," a median sternotomy was performed and the heart exposed. Heparin $(5,000 \mathrm{U})$ was infused intravenously to minimize intracoronary coagulation. The aorta, pulmonary artery, pulmonary veins, superior and inferior vena cavae, and the azygous vein were ligated and the heart removed. To further minimize intracoronary coagulation, the coronary ostia were cannulated and flushed with a heparinized saline solution $(20 \mathrm{U} / \mathrm{mL})$. The warm contrast-gelatin solution was then selectively infused into each coronary ostia using a syringe and a flanged catheter. The infusion was continued until the perforating vessels were filled by contrast, as determined by visual inspection. The specimen was placed in a cold $4 \%$ paraformaldehyde bath and refrigerated overnight to allow the gelatin to solidify.

\section{Image Acquisition and Preparation}

To stabilize the canine heart during the acquisition of image data, it was pierced with a pair of positioning rods and suspended within a plexiglass container (Fig 1). A sequence of contiguous transaxial computed tomography (CT) images were obtained from apex to base using spiral CT (Somatom Plus S, Siemens Medical Systems Inc, Iselin, $\mathrm{NJ}$ ). These images were obtained with 1-mm-slice thickness and no interspace gaps. CT was used rather than magnetic resonance imaging (MRI) because of its lower costs, ease of use with this model, and analogy of CT data to MRI volumetric data sets. The image sets were stored on optical disks.

The 159 individual CT images were postprocessed with the menu-driven software package, ANALYZE, and an imaging workstation (SparcStation 2, Sun Microsystems, Mountain View, CA). Each individual transaxial slice was reduced in size from 512- $\times 512-\times 1$-pixel to $256-\times 256-\times$ 1-pixel and scaled to an output maximum pixel intensity of 255. Each image was converted from 16 bits per pixel to 8 bits per pixel. Semiautomatic segmentation of the individual 2D CT images was used to remove the extracoronary structures (ie, positioning rods, myocardium, and plexiglass container). The resulting 116 eight-bit transaxial CT images depicting the coronary arteries were converted to binary data. Algorithms were created with a software development package, PV Wave (Precision Visuals Inc, Boulder, CO), to calculate the principal axis and centroid of the volumetric data set. A rotation matrix describing the conversion of the volumetric data set to a coordinate system defined by the principal axes was then calculated.

\section{Reformation of the Volumetric Image Data}

The rotation matrix, describing the spatial reformation of the original data set to an orthogonal reference system defined by the principal axes of the coronary artery tree, was used to reformat the original 8-bit dataset. Using ANALYZE, the 8-bit volumetric dataset was converted to the reference coordinate system described by the principal axis. The reformatted image volume was then projected using a maximum-intensity projection volume-rendering algorithm to display a panoramic cylindrical projection.

\section{Conversion to a Polar Coordinate System}

Because the data points describing the coronary anatomy are projected to a sphere with this panoramic rendering technique, certain scene components are necessarily distorted. The software development package, IDL, was used to generate additional mapping projections of the volumetric dataset. IDL requires polar coordinates as input to the mapping algorithms. Therefore, the orthogonal coordinates defining the coronary arteries were converted to polar coordinates.

A second binary file describing the coronary anatomy was created from the reformatted 8-bit dataset using segmentation algorithms and ANALYZE. The surface of the canine heart, defined by the coronary arteries, was assumed to approximate a sphere. Algorithms to calculate the representative latitude and longitude of each binary pixel (each pixel describing a portion of the coronary tree) were implemented. The longitudinal coordinate of each pixel defining the coronary arteries was defined as the angle (in radians) formed by a line passing through the centroid of the volume 
Fig 1. A plexiglass container constructed to stabilize the canine heart during imaging. The positioning rods are inserted through the myocardium at right angles. The heart is then placed within the container and the container placed on its side during image acquisition.

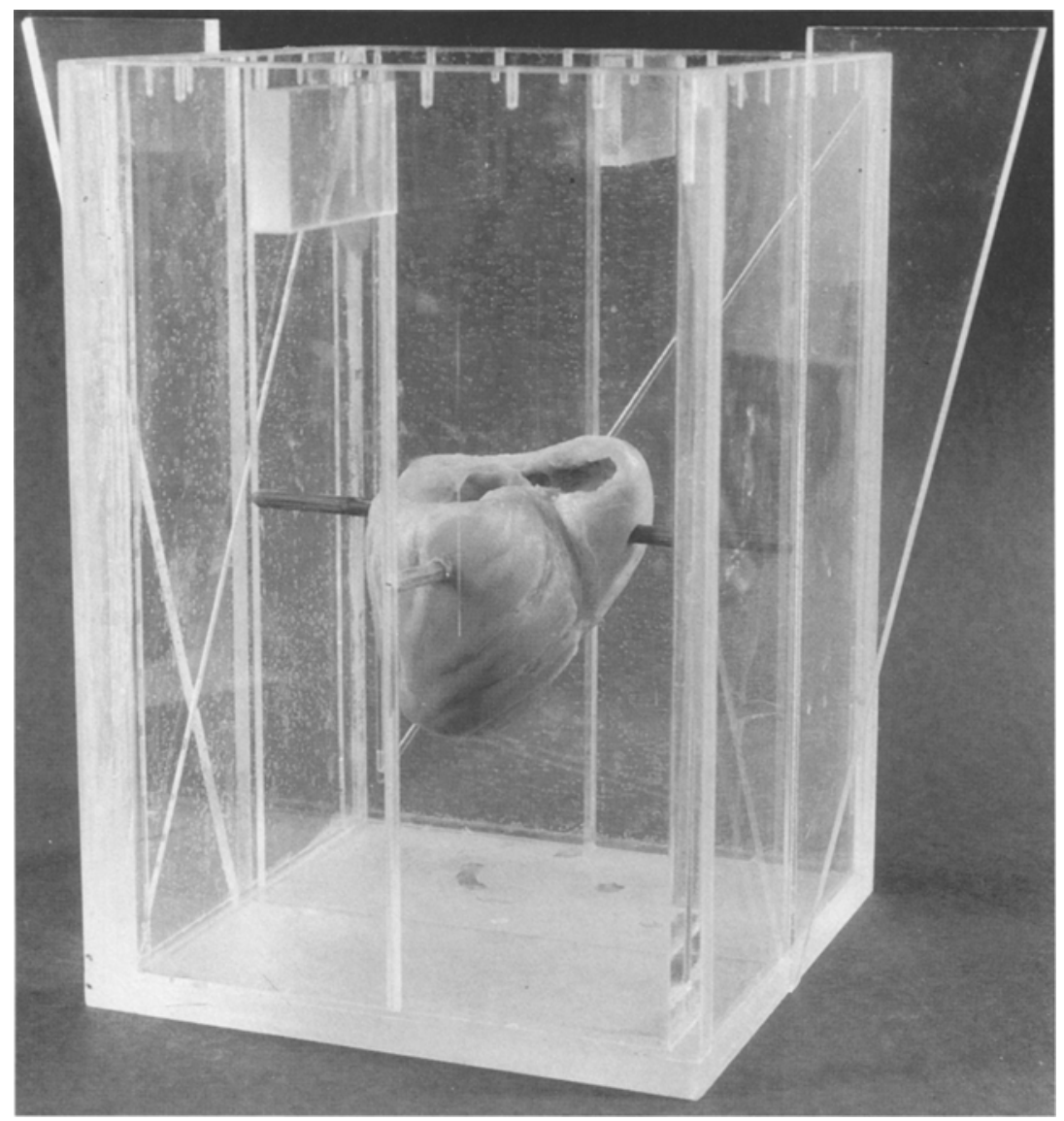

and the data point and a plane perpendicular to the principal axis and passing through the centroid. Pixels lying in the transverse plane intercepting the centroid were assigned a longitude of zero. Pixels lying between this transverse plane and the apex were assigned a negative value, and conversely, pixels lying between this transverse plane and the base were assigned a positive value. Similarly, the latitude coordinates were calculated by arbitrarily defining a line with a latitude of zero. The angle (in radians) formed by a plane formed by this line and the principal axis and a line extending from the centroid to the data point was determined. The latitude and longitude coordinates saved to a file.

The file containing these latitude and longitude coordinates is then used by IDL's built-in mapping algorithms. The IDL mapping algorithms (ie, Mercator, Mollwiede, conical, azimuthal, sinusoidal, cylindrical, Lambert, orthographic) were then used to display the coronary artery tree from a user defined oblique orientation. ${ }^{12}$

\section{RESULTS}

Using ANALYZE, both orthogonal (Fig 2) and cylindrical or panoramic representation of the canine coronary anatomy were created using a maximum-intensity projection volume ren- dering technique (Fig 3). This cylindrical projection was created from 116 individual 8-bit CT images reformatted to an orthogonal reference system defined by the principal axis of the canine heart. The spatial distortion of some scene components in this cylindrical projection is a consequence of the geometry of the mapped object. The spatial characteristics on the surface of certain 3D objects, such as a sphere, can only be approximated in the $2 \mathrm{D}$ representation.

The spatial orientation of each pixel in a volume of CT data is defined by Cartesian coordinates (ie, X, Y, Z). In addition, each pixel is further defined by the amount of information it contains (eg, binary, 8 bit, or 16 bit). The software development package, IDL, contains multiple mapping algorithms. These mapping algorithms require polar coordinates as input necessitating conversion of the individual data points in the $116 \mathrm{CT}$ images to their respective latitude and longitude equivalents. Further- 


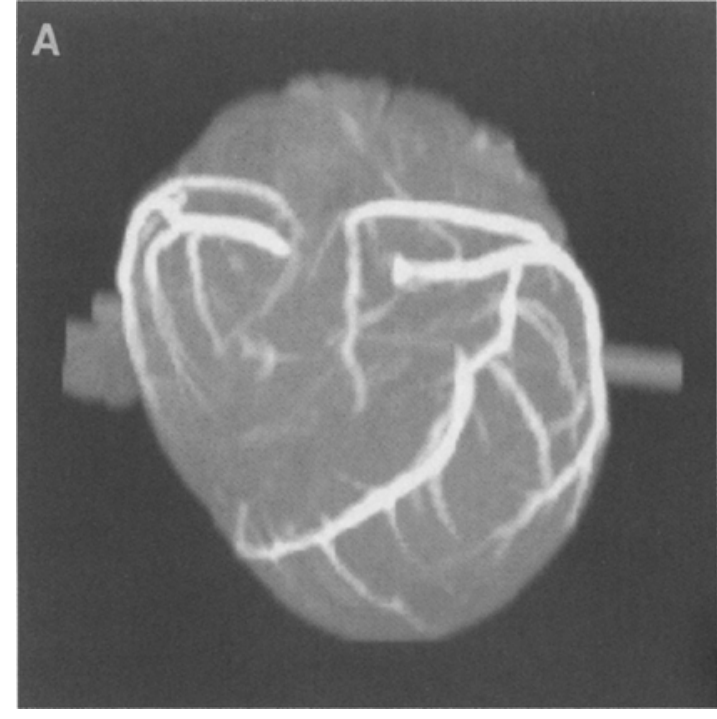

Fig 2. The orthogonal representations of the image volume are displayed. The images were created with ANALYZE using a maximum-intensity volume-rendering technique.

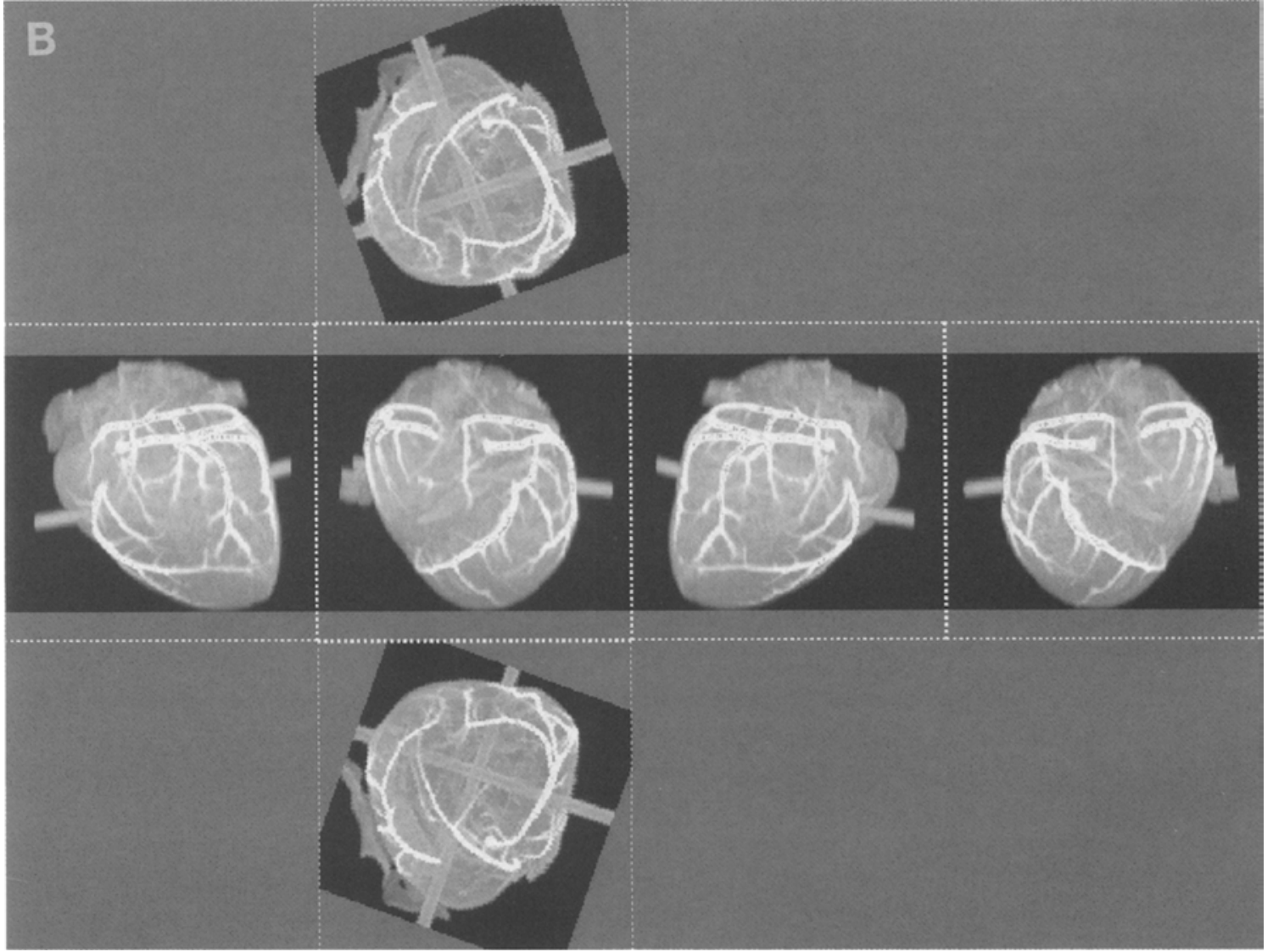

more, each coordinate is mapped as a binary pixel forcing the loss of resolution across the epicardial surface. Therefore, extracoronary structures were removed using segmentation and the remaining pixels depicting the coronary arteries were converted to binary format. After converting the orthogonal pixel positions to polar coordinates, the IDL mapping algorithms generated maps of coronary anatomy from multiple oblique orientations (Figs 4 and 5). 


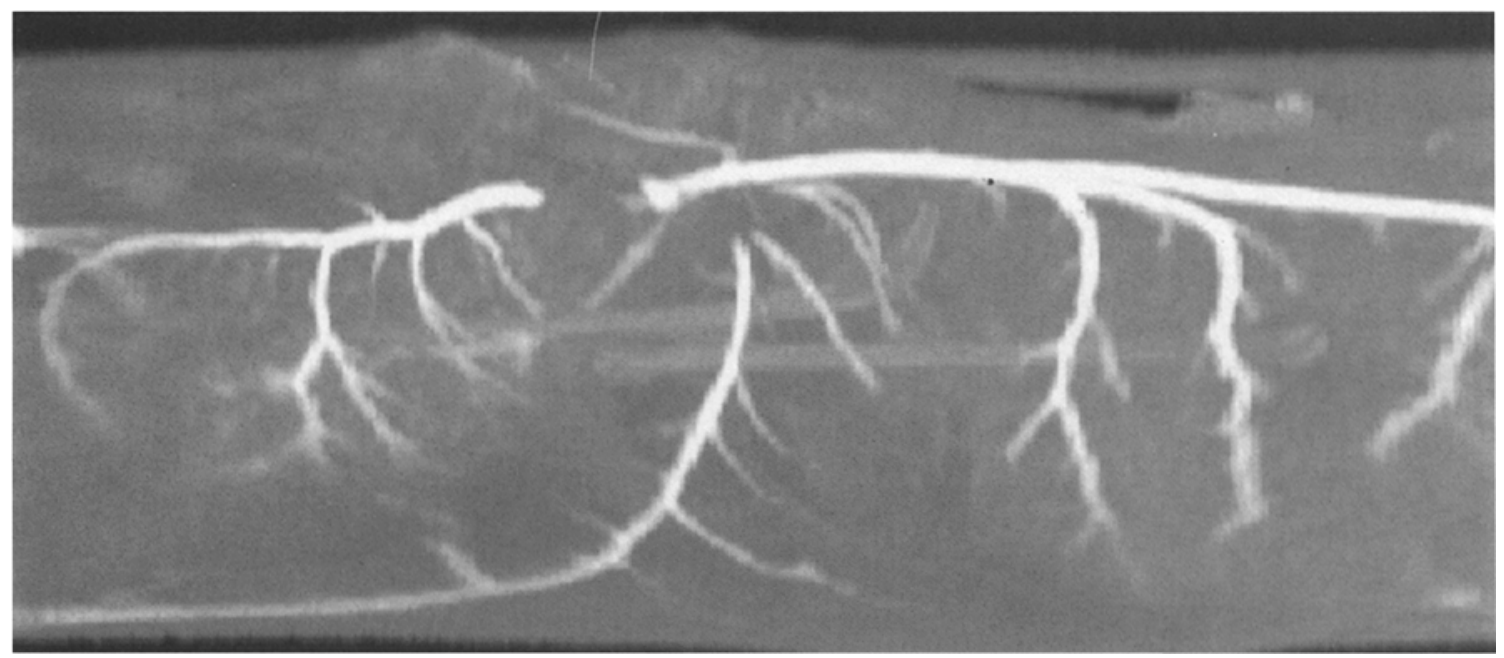

Fig 3. A panoramic rendering of the image volume using ANALYZE. This projection is generated using a maximum-intensity volume-rendering technique and a cylindrical projection process. The apparent defect in the left-anterior descending artery results from poor ex vivo injection of the contrast material.

\section{DISCUSSION}

Accurate identification of the epicardial arterial tree is necessarily subject to varied interpretations because of the superimposition of adjacent structures and the requirement for visual integration of multiple images. Conventional $3 \mathrm{D}$ reformation techniques may obscure scene components when viewed from certain orthogonal orientations (Fig 2). To overcome this deficiency, cartographic techniques may be used to display 3D volumes as 2D maps. These difficulties, as well as a recognition of the similarities between image analysis and cartography prompted this attempt at cartographic display of coronary anatomy. A variation of this principle was previously appreciated in the postmortem analysis of coronary anatomy. ${ }^{13}$ Our methods used computed tomographic data, but the technique is intuitively adaptable to other imaging modalities whose datasets are intrinsically volumetric, ie, echocardiography ${ }^{14,15}$ and MRI angiography.

Previous reports of 3D reformation of the coronary arterial tree have relied on the use of a Dynamic Spatial Reconstructor. ${ }^{16,17}$ These reformations have been generated by radially projecting each transverse slice from the center of the image slice (which is coincident with the intercept of the long axis of the left-ventricle chamber and the short-axis cross-sectional plane through the left ventricle) to an imaginary cylinder so as to create a polar display with the vertical dimension corresponding to transverse slice number and the horizontal dimension corresponding to the angle around the volume image. These reformatted images are analogous to cylindrical projection of the earth's surface. The determination of the long axis of the left-ventricle chamber is necessarily subjective with this method.

Certain characteristics are unique to any 3D object, eg, principal axes and the centroid. Rather than rely on the arbitrary identification of a central axis from which maps can be generated, these physical characteristics can be used to reformat the data volume. The principal axis is a line passing through the centroid which is equidistant from the points on the surface of the volume. In the example cited, the principal axis of the reformatted volume approximated the horizontal axis. Because the panoramic rendering algorithms are restricted to the vertical axis, it was necessary to convert the dataset from its instrument-specific coordinate system to a global coordinate system in which the principle axis approximated the ordinate. This was accomplished by rotating the volume 90 degrees with ANALYZE.

Application of cartographic methods to this type of medical imaging relies on a number of assumptions. The ability of the observer to accurately identify the coronary anatomy and 

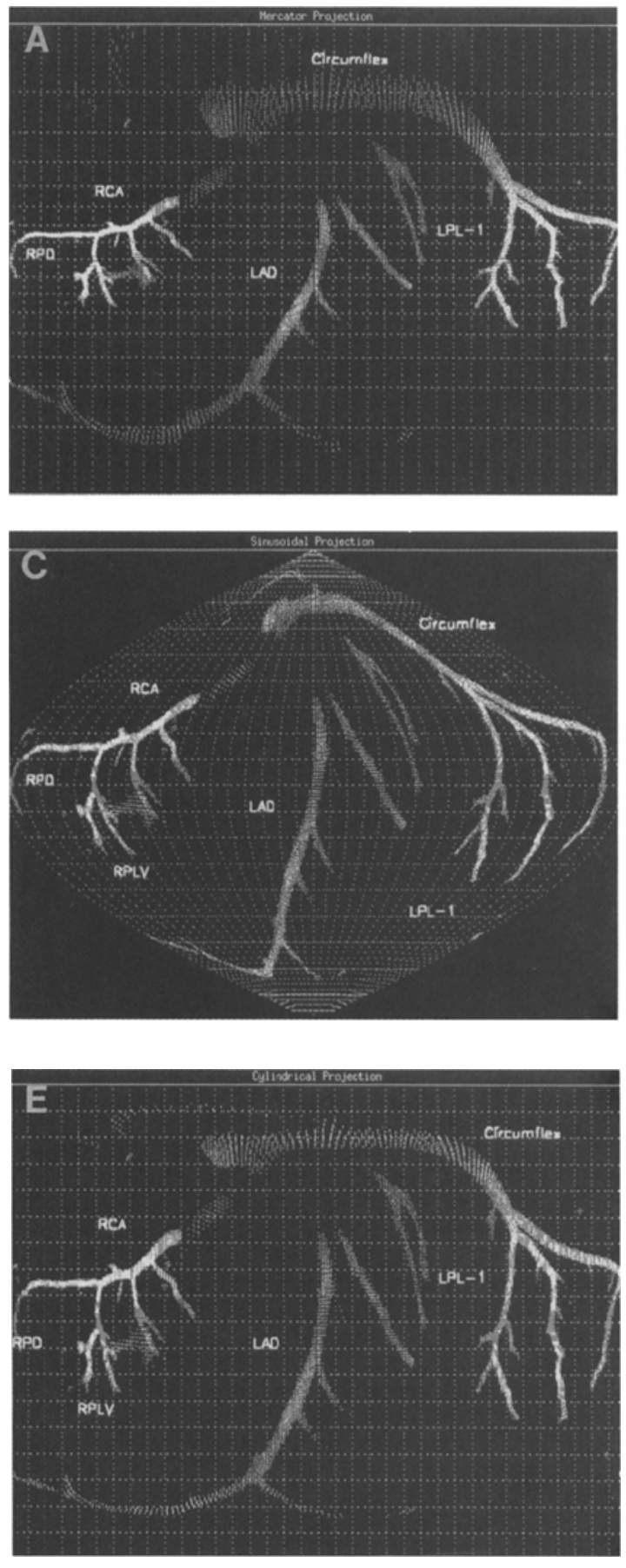

the limits of segmentation techniques make the conversion of the dataset to binary format and the accuracy of the subsequently reformatted images subject to error. The marked disparity in density between the contrast-filled vessels and the adjacent structures minimizes any error
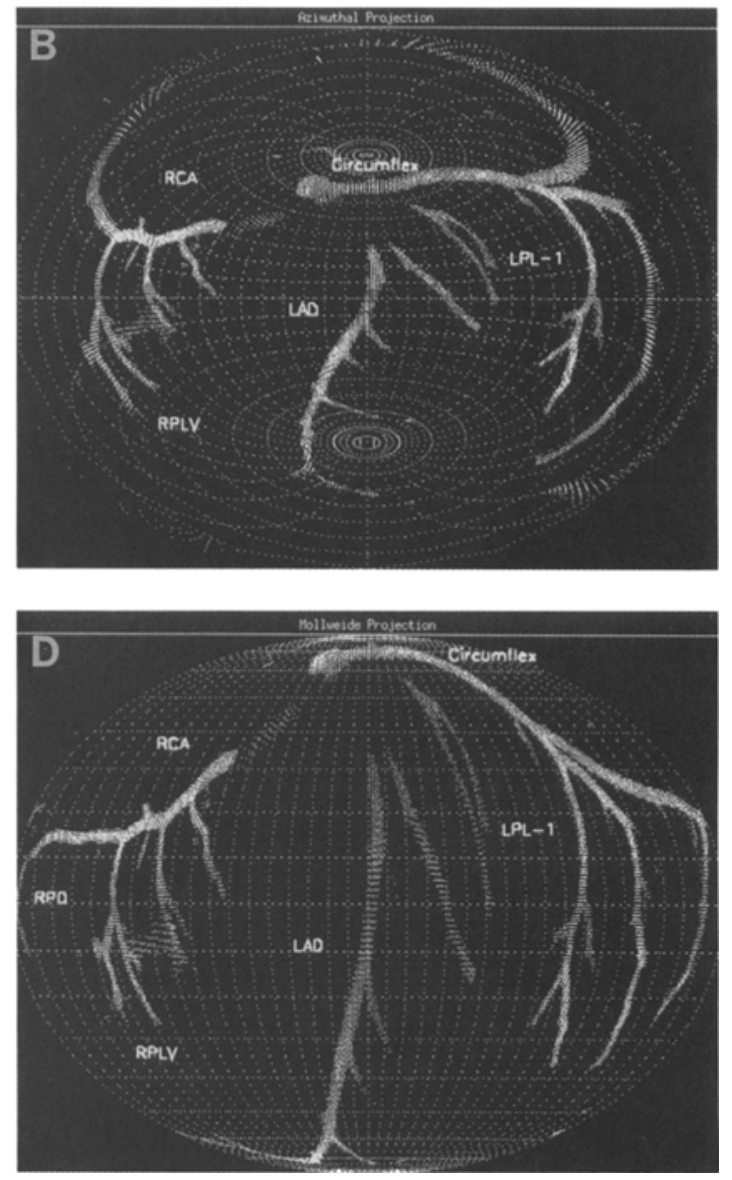

Fig 4. A demonstration of the variety of mapping projections available with the IDL software development package. The Mercator (A), azimuthal (B), sinusoidal (C), Mollwiede (D), and cylindrical (E) projections are shown.

associated with segmenting. Also, the conversion of Cartesian coordinates to polar coordinates necessarily assumes that the epicardial anatomy approximates a sphere. Furthermore, the perception of depth is lost because penetrating vessels are projected to the surface. 

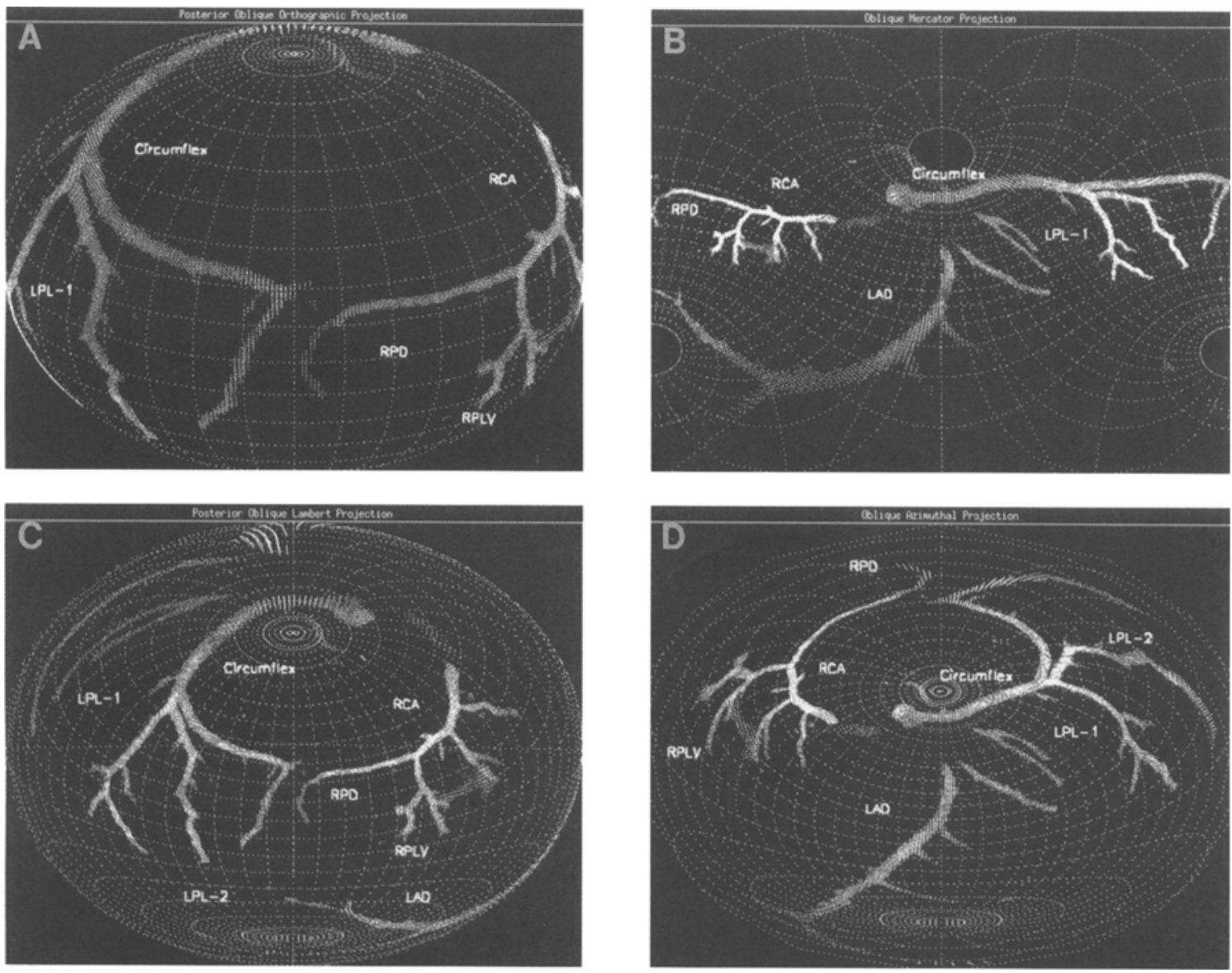

Fig 5. The IDL software development package allows creation of mapping projections from user-defined oblique reference angles. Shown are the posterior-oblique orthographic projection (A) with the angle of elevation set to 30 degrees; the oblique Mercator projection (B) with the angle of elevation set to 30 degrees; the posterior-oblique Lambert projection (C) with the angle of elevation set to 30 degrees; and the oblique azimuthal projection (D) with the angle of elevation set to 45 degrees.

\section{CONCLUSIONS}

Images obtained by cartographic projection methods clarify the relative position of the branches of the coronary arterial tree and obviate the need to mentally integrate multiple $2 \mathrm{D}$ images to obtain a $3 \mathrm{D}$ representation of this complex anatomic surface structure. These mapping projections offer the advantage of viewing 3D structure in a 2D plane and possibly clarifying anatomic structure obscured by superimposed anatomic detail. The inherent geometric distortion with any single projection and with the development of self-contained interactive imaging workstations, as well as with software packages for multidimensional medical image analysis and display has provided an opportunity to determine how beneficial 3D methods are in the evaluation of coronary anatomy.

\section{ACKNOWLEDGMENT}

We thank Dr Richard Robb for use of ANALYZE software (Mayo Biomedical Imaging Resource in Rochester, MN); Roberta L Yoffie for CT scanning technical assistance; Laura Brewer and Linda Montague for manuscript preparation; and Gulab Bhatia for software that calculated mass properties and principal axes.

\section{REFERENCES}

1. Alfidi RJ, Masaryk TJ, Haacke EM, et al: MR angiography of peripheral, carotid, and coronary arteries. AJR 149:1097-1109, 1987
2. Paulin S, von Schulthess GK, Fossel E, Krayenbuehl HP. MR imaging of the aortic root and proximal coronary arteries. AJR 148:665-670, 1987 
3. Manning WJ, Li W, Boyle NG, Edelman RR. Fatsuppressed breath-hold magnetic resonance coronary angiography. Circulation 87:94-104, 1993

4. Manning WJ, Li W, Edelman RR. A preliminary report comparing magnetic resonance coronary angiography with conventional angiography. N Engl J Med 328:828832,1993

5. Greenhood D: Mapping. Chicago, IL University of Chicago Press, 1964, pp 113-171

6. Pearson, F Jr: Map Projections: Theory and Applications. Boca Raton, FL, CRC Press, 1990, pp 347-358

7. Kaleps I, Clauser CE, Young JW, et al: Investigation into the mass distribution properties of the human body and its segments. Ergonomics 27:1225-1237, 1984

8. Alpert NM, Bradshaw JF, Kennedy D, Correia JA: The principal axes transformation - A method for image registration. J Nucl Med 31:1717-1722, 1990

9. Muehrcke PC: Map Use: Reading, Analysis, and Interpretation (ed 2). Madison, WI, JP Publications, 1986, pp 457-474

10. Robb RA: Data analysis, image processing, and display: Capabilities required for effective visualization and analysis of multi-modality, multidimensional biomedical images, in: Vannier MW, Yates RE, Whitestone JJ (eds): Electronic Imaging of the Human Body. Wright Patterson
AFB, OH; Crew System Ergonomics Information Analysis Center 1993:160-165.

11. Haskel EJ, Torr SR, Day KC, et al: Prevention of arterial reocclusion after thrombolysis with recombinant lipoprotein-associated coagulation inhibitor. Circulation 84 : 821-827, 1991

12. Snyder JP, Voxland PM: An Album of Map Projection, US Geological Survey Professional Paper 1453, Washington DC Government Printing Office, 1989

13. Schlesinger M: An Injection plus dissection study of coronary artery occlusions and anastomosis. Am Heart J 15:528-568, 1938

14. Belohlavek M, Foley DA, Gerber TC, et al: Threeand four-dimensional cardiovascular ultrasound imaging: A new era for echocardiography. Mayo Clinic Proc 68:221240, 1993

15. Greenleaf JF, Belohlavek $M$, Gerber TC, et al: Multidimensional visualization in echocardiography: An introduction. Mayo Clinic Proc 68:213-220, 1993

16. Liu Y-H, Bahn R, Ritman E: Myocardial volume perfused by coronary artery branches. Invest Rad 27:302307,1992

17. Liu Y-H, Bahn R, Ritman E: Myocardial volume perfused by coronary artery branches-a three-dimensional $\mathrm{x}$-ray CT evaluation of human cadaver hearts. Int J Card Imaging 8:95-101, 1992 